\title{
Promoting Photochromism on Flavylium Derived 2-Hydroxychalcones in Aqueous Solutions by Addition of CTAB Micelles
}

Raquel Gomes, A. Jorge Parola, César A. T. Laia, Fernando Pina

\section{Supplementary material}

1. Kinetics of the network of chemical reactions of

7-(N,N-diethylamino)-4'-hydroxyflavylium in water

2. How do CTAB micelles affect the equilibria of the flavylium network 


\section{Kinetics of the network of chemical reactions of}

At the equilibrium, in acidic to slightly basic media, Fig. S1A, the system can be described by eq. (1), because the flavylium cation $\mathrm{AH}^{+}$and the trans-chalcone $\mathrm{Ct}$ behave as a single acid base reaction. ${ }^{1}$ In other words, the amount of the remaining species reported in Scheme 1 can be neglected at the equilibrium. However, these species control the kinetic processes.
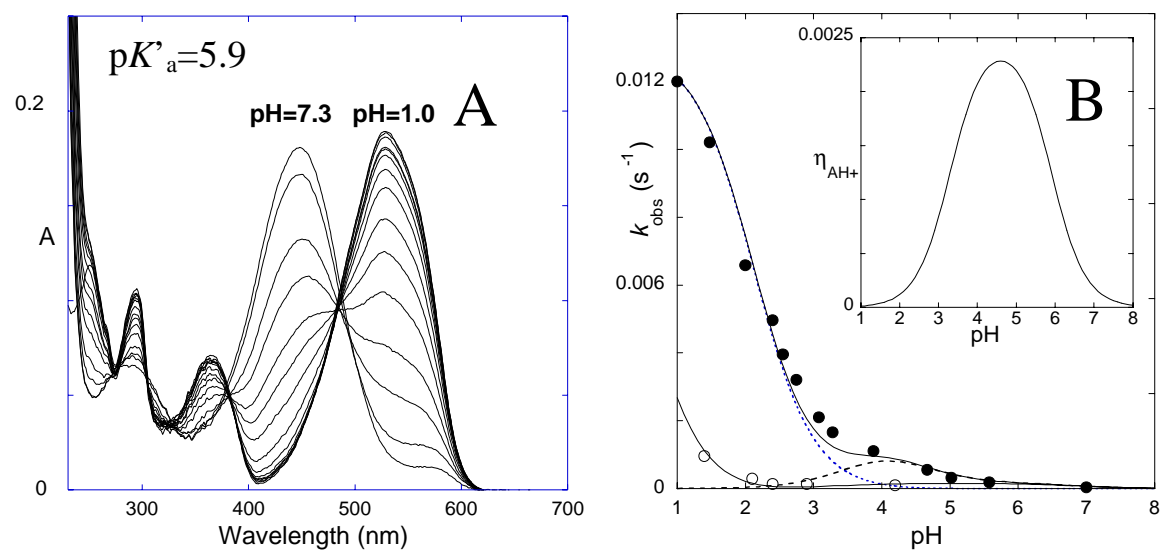

Fig. S1. Thermodynamic (A) and kinetics (B) of 7-(N,N-diethylamino)-4'-hydroxyflavylium $6.7 \times 10^{-6} \mathrm{M}$. A: $\mathrm{pH}$ dependent absorption spectra at room temperature after 1 day in the dark; B: black circles; reaction rate of $\mathrm{AH}^{+} / \mathrm{A}$ formation from chalcones as a function of $\mathrm{pH} ;{ }^{2}$ open circles correspond to the experimental rates in the presence of CTAB micelles; fitting was achieved by means of eq. (3); inset: efficiency, $\eta_{\mathrm{AH}^{+}}$, of the photochemical reaction.

As reported in previous work, ${ }^{2}$ the flavylium $\mathrm{AH}^{+}$, and the chalcones can be interconverted through $\mathrm{pH}$ changes, the respective rates being shown in Fig. S1B. The observed rate constants can be accounted for by considering the superposition of two components, one involving the species $\mathrm{AH}^{+} / \mathrm{Ct}$, eqs. (S1) and (S2), (traced line) and the other, the species $\mathrm{AH}^{+} / \mathrm{Ct}^{+}$, eq. (S3), (pointed line).
$\mathrm{AH}^{+} \rightleftharpoons \mathrm{A}+\mathrm{H}^{+}$
$K_{\mathrm{a}}=10^{-6.6}$ 


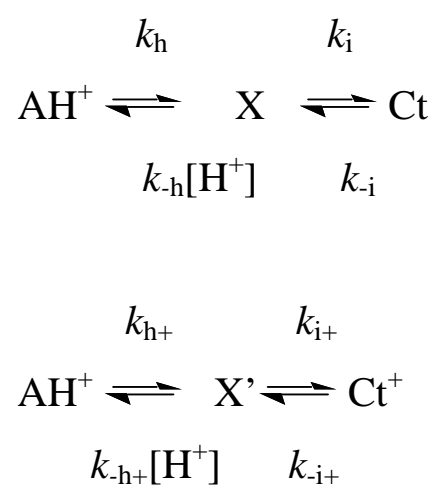

where $\mathrm{X}$ and $\mathrm{X}^{\prime}$ are the intermediate states between $\mathrm{AH}^{+}$and $\mathrm{Ct}$ species, constituted by hemiketal $\left(\mathbf{B}_{2}\right)$ and cis-chalcone $(\mathbf{C} \mathbf{c})$ in their unprotonated and/or protonated forms. The kinetic treatment of this system permits to calculate the rate constants involved in eqs. (S2) and (S3), as follows; $k_{\mathrm{h}}=1.5 \times 10^{-4} \mathrm{~s}^{-1} ; k_{\mathrm{i}}=1.0 \times 10^{-2} \mathrm{~s}^{-1} ; k_{\mathrm{i}} / k_{\mathrm{h}}=5.0 \times 10^{-4} \mathrm{M} ; k_{\mathrm{i}+} / k_{\text {- }}$ ${ }_{\mathrm{h}+}=7.5 \times 10^{-3} \mathrm{M} ; k_{\mathrm{-i}+}=1.3 \times 10^{-2} \mathrm{~s}^{-1}$.

The present compound does not exhibit measurable photochemistry in water. ${ }^{2}$ The lack of significant photochemistry in chalcones possessing an amino group was previously reported, and attributed to the formation of a highly polar state on the $S_{1}$ hypersurface, presumably a twisted intramolecular charge transfer state (TICT) ${ }^{3}$ or another ICT mechanism. Such mechanism implies the presence of an extra reaction coordinate at the chalcone singlet excited state, which would quench the trans-cis photoisomerization reaction (as well as its fluorescence) giving rise to very low photoisomerization quantum yields. Nevertheless, kinetic reasons may also contribute to the absence of measurable photochemistry in water, when eq. (S4) is introduced.

$\eta_{A H^{+}}=\frac{\mathrm{K}_{\mathrm{a}}^{\prime}}{\left[\mathrm{H}^{+}\right]+\mathrm{K}_{\mathrm{a}}^{\prime}} \cdot \frac{\mathrm{k}_{-\mathrm{h}}\left[\mathrm{H}^{+}\right]}{\mathrm{k}_{\mathrm{i}}+\mathrm{k}_{-\mathrm{h}}\left[\mathrm{H}^{+}\right]}=\frac{10^{-5.9}}{\left[\mathrm{H}^{+}\right]+10^{-5.9}} \cdot \frac{2 \times 10^{3}\left[\mathrm{H}^{+}\right]}{1+2 \times 10^{3}\left[\mathrm{H}^{+}\right]}$

This expression is a measure of the $\mathrm{pH}$ dependent efficiency of $\mathrm{AH}^{+}$formation upon irradiation of $\mathrm{Ct}^{4}{ }^{4}$ The first term regards the mole fraction of $\mathrm{Ct}$ available at a certain $\mathrm{pH}$ value, and the second term accounts for the efficiency of $\mathrm{AH}^{+}$formation from the intermediate X. Substitution of the values ${ }^{2}$ of the respective constants in eq. (S4) (see inset of Fig. S1B) shows indeed low values for $\eta_{\mathrm{AH}_{+}}$, explaining why this system does not give rise to a significant photochromism in water. 


\section{How do CTAB micelles affect the equilibria of the flavylium network}

The situation is dramatically changed when CTAB micelles (monomer $5 \times 10^{-3} \mathrm{M}$ ) are added to aqueous solutions of 7-(N,N-diethylamino)-4'-hydroxyflavylium, Fig. S2. Addition of CTAB micelles to a solution of $\mathrm{Ct}^{2-}$ leads to a red shifted absorption band indicating the existence of an interaction micelle-unprotonated chalcone, Fig. S2A. This process occurs during the mixing time as no further spectral changes were observed. This interaction can be accounted for by the electrostatic attraction between CTAB micelles and $\mathrm{Ct}^{2-}$. However, the electrostatic interaction is not the only parameter to take into account, because the neutral chalcone, $\mathrm{Ct}$, also leads to spectral variations upon CTAB micelles addition, forming an adduct herein identified as Ct-CTAB, Fig. S2B. Even more interesting is the interaction of $\mathrm{Ct}^{+}$, with $\mathrm{CTAB}$ micelles, Fig. S2C. The protonated chalcone can be obtained from $\mathrm{Ct}$ upon a $\mathrm{pH}$ jump: while in the absence of CTAB micelles $\mathrm{Ct}^{+}$leads to $\mathrm{AH}^{+}$, in the presence of the micelles leads to the adduct Ct-CTAB. This result shows that the interaction between $\mathrm{Ct}$ and the micelle is sufficiently strong to displace the equilibrium towards adduct formation. On the other hand, at very acidic $\mathrm{pH}$ values $\mathrm{AH}^{+}$is stable even in the presence of micelles, and no spectral variations are detected, indicating the lack of interaction with the micelles, as expected from the electrostatic repulsion.

In order to get more insight on 7-(N,N-diethylamino)-4'-hydroxyflavylium network interaction with $\mathrm{CTAB}$ micelles in the acidic region, the following experiments were carried out: $1 \mathrm{ml}$ of $\mathrm{Ct}^{2-}$ at $\mathrm{pH} 12$ was added to $2 \mathrm{ml}$ of a solution containing acid and buffer to give the desired final $\mathrm{pH}$ value together with $[\mathrm{CTAB}]=5 \times 10^{-3} \mathrm{M}$. This procedure allows instantaneous formation of the transient $\mathrm{Ct}^{+}$. As can be confirmed by Fig. S3, the protonated chalcone is involved in a partition equilibrium between micelles $(\mathrm{Ct}-\mathrm{CTAB})$ and bulk water. Taking into account that $\mathrm{p} K_{\mathrm{Ct}+}=4.5,{ }^{2}$ in the $\mathrm{pH}$ range of this experiment formation of $\mathrm{Ct}^{+}$is expected. The partition of $\mathrm{Ct}^{+}$and $\mathrm{Ct}$ is finished before the time needed to run a spectrum, $c a .30 \mathrm{~s}$. In other words, $\mathrm{Ct}^{+}$goes very fast inside the micelle to form Ct-CTAB and a pseudo-equilibrium is attained with $\mathrm{p} K_{\mathrm{obs}}=1.1$. 

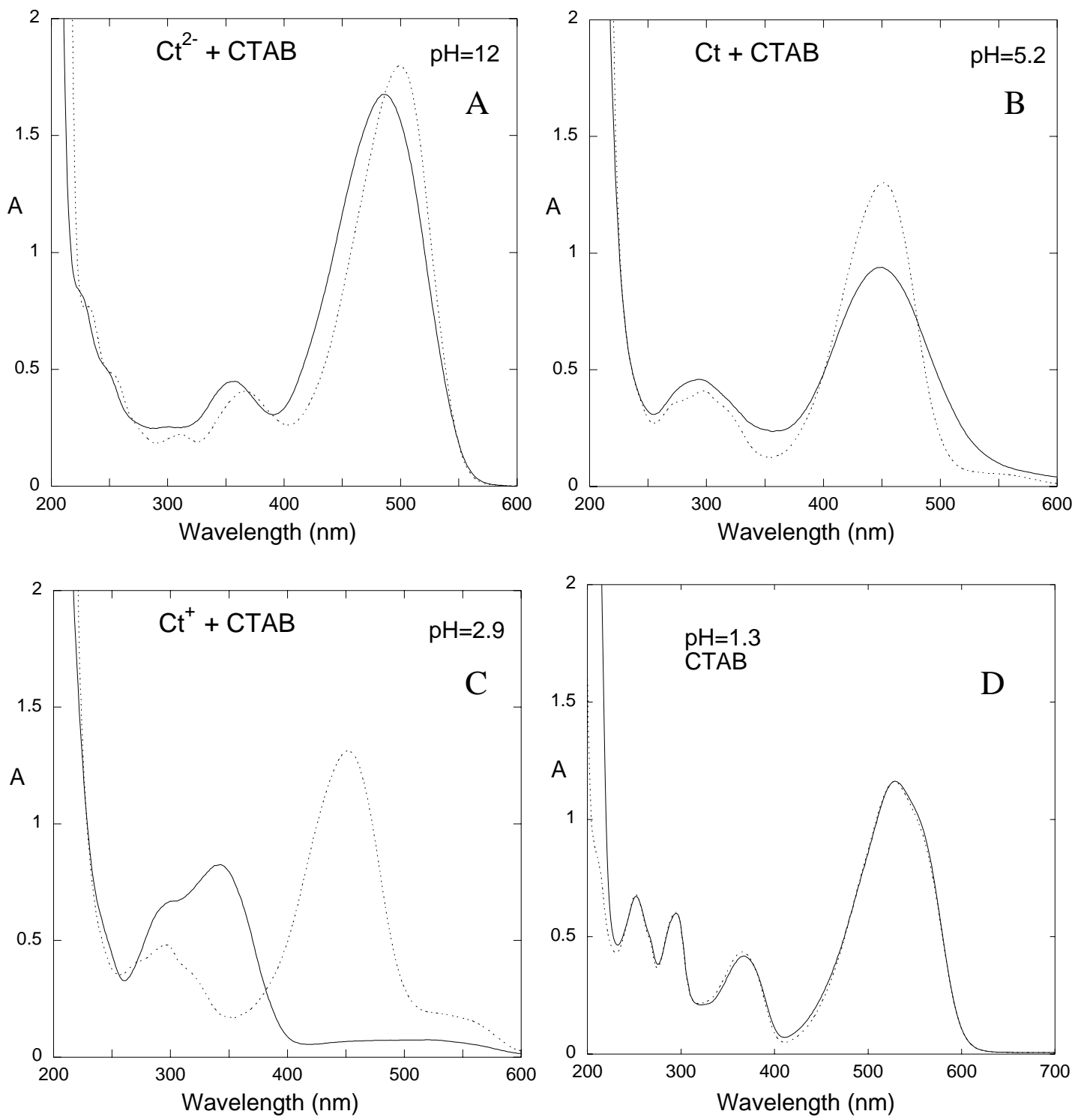

Fig. S2. Spectral variations of trans-chalcone solutions at $\mathrm{pH} 12, \mathrm{Ct}^{2-},(\mathrm{A})$, at $\mathrm{pH}=5.2, \mathrm{Ct}$, (B) and 2.9, $\mathrm{Ct}^{+}$, (C) and flavylium cation, $\mathrm{AH}^{+}$, (D) of 7-(N,N-diethylamino)-4'-hydroxyflavylium (full line) obtained immediately (time scale of the spectrophotometer) upon addition of CTAB (pointed lines). $\left[\mathrm{AH}^{+}\right]=3.8 \times 10^{-5}$ $\mathrm{M}$ and $[\mathrm{CTAB}]=5 \times 10^{-3} \mathrm{M}$. 


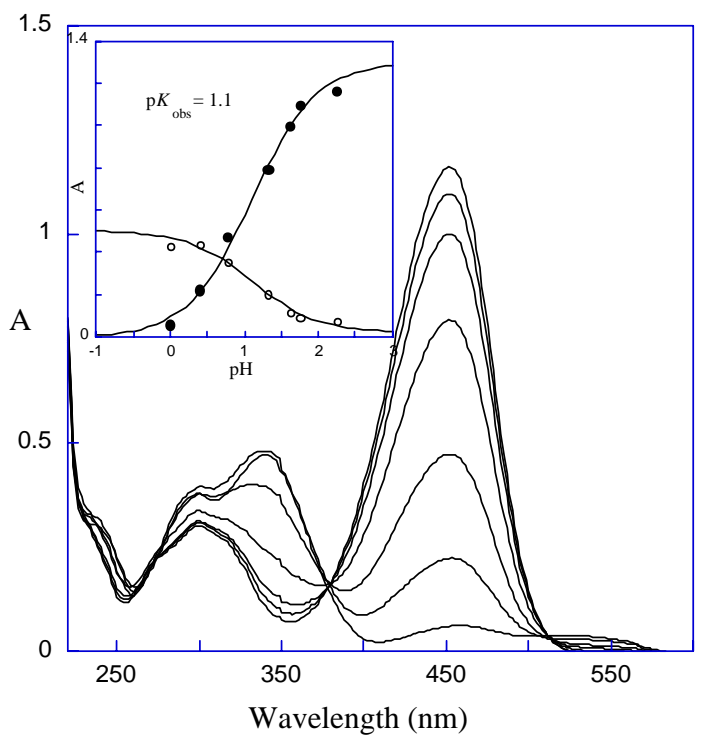

Fig. S3. Spectral variations of trans-chalcone solutions of 7-(N,N-diethylamino)-4'-hydroxyflavylium

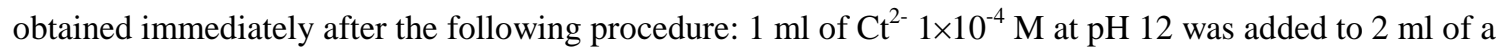
solution containing acid and buffer to give the desired final $\mathrm{pH}$ value and $\mathrm{CTAB} ;[\mathrm{CTAB}]_{\text {final }}=5 \times 10^{-3} \mathrm{M}$; inset - absorption at $450 \mathrm{~nm}$ (black circles) and at $350 \mathrm{~nm}$ (white circles) versus $\mathrm{pH}$, fitting was achieved with $\mathrm{p} K_{\mathrm{obs}}=1.1$.

While Fig. S3 accounts for a transient situation (pseudo-equilibrium), the $\mathrm{pH}$ dependent spectral variations reported in Fig. 1 correspond to the equilibrium, where the main species observed are $\mathrm{Ct}-\mathrm{CTAB}$ and $\mathrm{AH}^{+}$.

The data reported in Fig. S3 (pseudo-equilibrium) can be accounted for by the following set of equations, where only three are linearly independent:

$$
\begin{array}{ll}
\mathrm{Ct}^{+} \rightleftharpoons \mathrm{Ct}+\mathrm{H}^{+} & K_{\mathrm{Ct}+}=10^{-4.5} \\
\mathrm{Ct} \rightleftharpoons \mathrm{Ct}-\mathrm{CTAB} & K_{\mathrm{p} 1} \\
\mathrm{Ct}^{+} \rightleftharpoons \mathrm{Ct}^{+}-\mathrm{CTAB} & K_{\mathrm{p} 2} \\
\mathrm{Ct}^{+}-\mathrm{CTAB} \rightleftharpoons \mathrm{Ct}-\mathrm{CTAB}+\mathrm{H}^{+} & K_{\mathrm{Ct}+}{ }^{\mathrm{m}}=K_{\mathrm{Ct}+} K_{\mathrm{p} 1} / K_{\mathrm{p} 2} \\
& \\
C_{0}=[C t]+\left[C^{+}\right]+[C t-C T A B]+\left[C t^{+}-C T A B\right]=\left[C t^{+}\right]\left(\left(1+K_{p 2}\right)+\frac{K_{C^{+}}\left(1+K_{\mathrm{p} 1}\right)}{\left[H^{+}\right]}\right]
\end{array}
$$


$\chi_{C t^{+}}=\frac{\left[C t^{+}\right]}{C_{0}}=\frac{1}{1+K_{p 2}} \cdot \frac{\left[H^{+}\right]}{\left[H^{+}\right]+\frac{K_{C t^{+}}\left(1+K_{p 1}\right)}{1+K_{p 2}}}$

The inflection point of a $\chi_{C t^{+}}$versus $\mathrm{pH}$ is thus equal to $\frac{K_{C t^{+}}\left(1+K_{p 1}\right)}{1+K_{p 2}}=10^{-1.1}$ (see inset of

Fig. 1A) allowing to calculate:

$\frac{1+K_{p 1}}{1+K_{p 2}}=10^{3.4}$

On the other hand, at the equilibrium, eq. (1) should be considered and

$C_{0}=\left[A H^{+}\right]+[C t]+\left[C t^{+}\right]+[C t-C T A B]+\left[C t^{+}-C T A B\right]=\left[A H^{+}\right]\left(1+\frac{K_{a}^{\prime}}{\left[H^{+}\right]}+\frac{K_{p 1} K_{a}^{\prime}}{\left[H^{+}\right]}+\frac{K_{a}^{\prime}}{K_{C t^{+}}}+\frac{K_{p 2} K_{a}^{\prime}}{K_{C t^{+}}}\right)$

$$
\frac{\left[A H^{+}\right]}{C_{0}}=\frac{K_{C t^{+}}}{K_{C t^{+}}+K_{a}^{\prime}\left(1+K_{p 2}\right)} \frac{\left[H^{+}\right]}{\left[H^{+}\right]+\frac{K_{a}^{\prime}\left(1+K_{p 1}\right) K_{C t^{+}}}{K_{C t^{+}}+K_{a}^{\prime}\left(1+K_{p 2}\right)}}
$$

It is worth of note that eq. (S13) predicts the existence of a plateau for high proton concentration, since the second term equals unity. The existence of this plateau was confirmed through the experiment reported in Fig. 1B. To an equilibrated solution of $\mathrm{AH}^{+}(1 \mathrm{M} \mathrm{HCl})$ the micelle was added and the absorption spectra monitored as a function of time. The decrease in flavylium concentration is in agreement with the plateau predicted from eq. (S13).

$\frac{K_{C t^{+}}}{K_{C t^{+}}+K_{a}^{\prime}\left(1+K_{p 2}\right)}=0.91$ 
Using eqs. (S10) and (S14), the partition coefficients can be evaluated as well as the $\mathrm{p} K_{\mathrm{a}}$ of $\mathrm{Ct}^{+}$inside the micelle, eq. (S8):

$K_{p 1}=6280$

$K_{p 2}=1.5$

$p K_{C t+}{ }^{m}=0.9$

The $\mathrm{pH}$ dependent mole fraction distribution of $\mathrm{AH}^{+}$at the equilibrium, can now be calculated by means of eq. (S13). Identical distributions can be easily obtained for the other species at the equilibrium, Fig. $2 \mathrm{~A}$ of the main text. 
Eq. (3) was deduced according to Scheme 2:

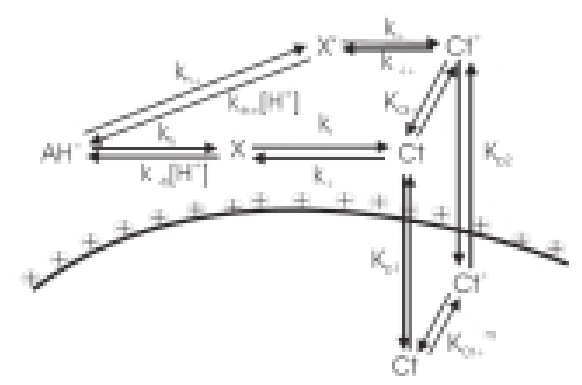

Scheme 2

The rate of $\mathbf{A H}^{+}$conversion to $\mathbf{C t - C T A B}$ and $\mathbf{C t} \mathbf{t}^{+}-\mathbf{C T A B}$ follows a first-order kinetics, which means that a great number of approximations may be done, despite the fact that many intermediate species are involved. First one must write the differential equations.

$$
\begin{aligned}
& \frac{d\left[A H^{+}\right]}{d t}=-\left(k_{h}+k_{h+}\right)\left[A H^{+}\right]+\left(k_{-h}[X]+k_{-h+}\left[X^{\prime}\right]\right)\left[H^{+}\right] \\
& \frac{d[X]}{d t}=k_{h}\left[A H^{+}\right]-\left(k_{-h}\left[H^{+}\right]+k_{i}\right)[X]+k_{-i}[C t] \\
& \frac{d\left[X^{\prime}\right]}{d t}=k_{h+}\left[A H^{+}-\left(k_{-h+}\left[H^{+}\right]+k_{i+}\right)\left[X^{\prime}\right]+k_{-i+}\left[C t^{+}\right]\right. \\
& \frac{d\left([C t]+\left[C t^{+}\right]+[C t-C T A B]+\left[C t^{+}-C T A B\right]\right)}{d t}=k_{i}[X]+k_{i+}\left[X^{\prime}-k_{-i}[C t]-k_{-i+}\left[C t^{+}\right]\right.
\end{aligned}
$$

Defining

$$
\left.C_{0}=[C t]+\left[C t^{+}\right]+[C t-C T A B]+\left[C t^{+}-C T A B\right]=\left[C t^{+}\right]\left(1+K_{p 2}\right)+\frac{K_{C^{+}}\left(1+K_{p 1}\right)}{\left[H^{+}\right]}\right]
$$

One obtains 


$$
\begin{aligned}
& \chi_{C t^{+}}=\frac{\left[C t^{+}\right]}{C_{0}}=\frac{1}{1+K_{p 2}} \cdot \frac{\left[H^{+}\right]}{\left.\left[H^{+}\right]+\frac{K_{C t^{+}}}{1+K_{p 2}}\right)} \\
& \chi_{C t}=\frac{[C t]}{C_{0}}=\frac{1}{1+K_{p 2}} \cdot \frac{K_{C^{+}}}{\left[H^{+}\right]+\frac{K_{C t^{+}}\left(1+K_{p 1}\right)}{1+K_{p 2}}}
\end{aligned}
$$

If the molar fractions are introduced in the set of differential equations, the following expressions are obtained:

$$
\begin{aligned}
& \frac{d[X]}{d t}=k_{h}\left[A H^{+}\right]-\left(k_{-h}\left[H^{+}\right]+k_{i}\right)[X]+k_{-i} \chi_{C t} C_{0} \\
& \frac{d\left[X^{\prime}\right]}{d t}=k_{h}^{+}\left[A H^{+}\right]-\left(k_{-h+}\left[H^{+}\right]+k_{i+}\right)\left[X^{\prime}\right]+k_{-i+} \chi_{C^{+}} C_{0} \\
& \frac{d\left(C_{0}\right)}{d t}=k_{i}[X]+k_{i+}\left[X^{\prime}\right]-\left(k_{-i} \chi_{C t}+k_{-i+} \chi_{C t^{+}}\right) C_{0}
\end{aligned}
$$

Now steady-state approximations are made for the intermediate species represented by $\mathbf{X}$ and $\mathbf{X}^{\prime}$, leading to:

$$
\begin{aligned}
& {[X]=\frac{k_{h}\left[A H^{+}\right]+k_{-i} \chi_{C t} C_{0}}{k_{-h}\left[H^{+}\right]+k_{i}}} \\
& {\left[X^{\prime}\right]=\frac{k_{h+}\left[A H^{+}\right]+k_{-i+} \chi_{C^{+}} C_{0}}{k_{-h+}\left[H^{+}\right]+k_{i+}}}
\end{aligned}
$$

After the substitutions, eqs. (S15) and (S24) give:

$$
\begin{aligned}
& \frac{d\left[A H^{+}\right]}{d t}=-\left(\frac{k_{h} k_{i}}{k_{-h}\left[H^{+}\right]+k_{i}}+\frac{k_{h+} k_{i+}}{k_{-h+}\left[H^{+}\right]+k_{i+}}\right)\left[A H^{+}\right]+\left(\frac{k_{-h} k_{-i} \chi_{C t}}{k_{-h}\left[H^{+}\right]+k_{i}}+\frac{k_{-h+} k_{-i+} \chi_{C t^{+}}}{k_{-h+}\left[H^{+}\right]+k_{i+}}\right)\left[H^{+}\right] C_{0} \\
& \frac{d\left(C_{0}\right)}{d t}=\left(\frac{k_{h} k_{i}}{k_{-h}\left[H^{+}\right]+k_{i}}+\frac{k_{h+} k_{i+}}{k_{-h+}\left[H^{+}\right]+k_{i+}}\right)\left[A H^{+}\right]\left(\frac{k_{-h} k_{-i} \chi_{C t}}{k_{-h}\left[H^{+}\right]+k_{i}}+\frac{k_{-h+} k_{-i+} \chi_{C t^{+}}}{k_{-h+}\left[H^{+}\right]+k_{i+}}\right)\left[H^{+}\right] C_{0}
\end{aligned}
$$


And therefore the observed rate constant would be:

$k_{\text {thermal }}=\frac{\left[H^{+}\right]}{\left[H^{+}\right]+K_{a}}\left(\frac{k_{h} k_{i}}{k_{-h}\left[H^{+}\right]+k_{i}}+\frac{k_{h+} k_{i+}}{k_{-h+}\left[H^{+}\right]+k_{i+}}\right)+\left(\frac{k_{-h} k_{-i} \chi_{C t}}{k_{-h}\left[H^{+}\right]+k_{i}}+\frac{k_{-h+} k_{-i+} \chi_{C^{+}}}{k_{-h+}\left[H^{+}\right]+k_{i+}}\right)\left[H^{+}\right]$

The factor $\left[\mathrm{H}^{+}\right] /\left(\left[\mathrm{H}^{+}\right]+\mathrm{K}_{\mathrm{a}}\right)$ appears in order to correct the $\left[\mathrm{AH}^{+}\right]$, due to the acid-base equilibrium of this species. It turns out the $\chi_{\mathrm{Ct}} \approx 0$ at all $\mathrm{pH}$ values therefore eq. (S28) can be approximated to eq. (3). 
Eqs. (4) and eqs. (5) were deduced according to Scheme 3:

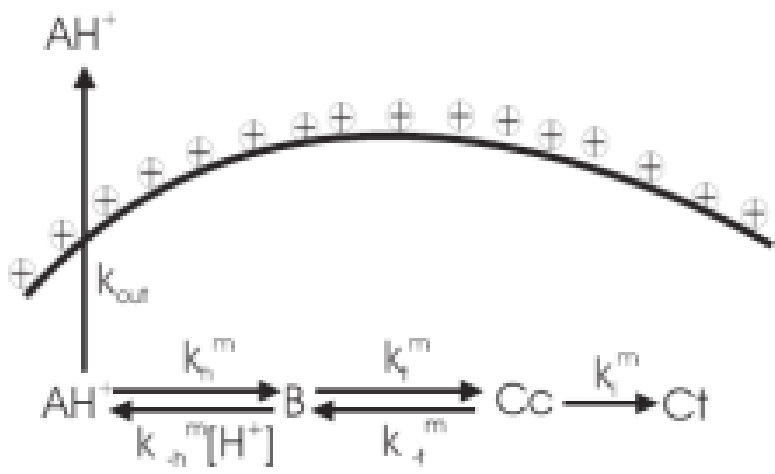

Scheme 3

The differential equations that describe the kinetics according to the scheme are:

$\frac{d[C t-C T A B]}{d t}=k_{i}^{m}[C c-C T A B$

$\frac{d[C c-C T A B]}{d t}=-\left(k_{i}^{m}+k_{-t}^{m}\right)[C c-C T A B]+k_{t}^{m}[B-C T A B]$

$\frac{d[B-C T A B]}{d t}=k_{-t}^{m}[C c-C T A B]-\left(k_{-h}^{m}\left[H^{+}++k_{t}^{m}\right)[B-C T A B]+k_{h}^{m}\left[A H^{+}-C T A B\right]\right.$

$\frac{d\left[A H^{+}-C T A B\right]}{d t}=k_{-h}^{m}\left[H^{+}[B-C T A B]-\left(k_{\text {out }}+k_{h}^{m}\right)\left[A H^{+}-C T A B\right]\right.$

$\frac{d\left[A H^{+}\right]}{d t}=k_{\text {out }}\left[A H^{+}-C T A B\right]$

Since Ct-CTAB and $\mathbf{A H}^{+}$are products, equations (S29) and (S33) are not necessary for the explanation of the observed rate constants. Therefore the set of equations is reduced to (S30), (S31) and (S32). In order to solve them, one can apply steady-state approximations for some of the intermediate products, namely B-CTAB and $\mathbf{A H}^{+}$CTAB. The experimental kinetics can be solved with biexponential functions, which 
gives two first order observed rate constants ( $k_{\text {flash }}{ }^{I}$ and $\left.k_{\text {flash }}{ }^{I I}\right)$. Now one can envisage Scheme 3 as two sequential steps. The first step occurs immediately after the photochemical production of Cc-CTAB, and can be attributed to the fast formation of the hemiketal species B-CTAB. The second step occurs on a much larger timescale, and deals with the production of $\mathbf{A H}{ }^{+}$and $\mathbf{C t - C T A B}$. Under such circumstances, it might be feasible to apply the steady-state approximation to all intermediate products, as long as their concentrations are always very low. If one makes such approximation, the following expressions are derived:

$$
\begin{aligned}
& {\left[A H^{+}-C T A B\right]=\frac{k_{-h}^{m}\left[H^{+}\right]}{k_{h}^{m}+k_{\text {out }}}[B-C T A B]} \\
& {[B-C T A B]=\frac{k_{-t}^{m}\left(k_{h}^{m}+k_{\text {out }}\right)}{k_{t}^{m}\left(k_{h}^{m}+k_{\text {out }}\right)+k_{\text {out }} k_{-h}^{m}\left[H^{+}\right.}[C C-C T A B]}
\end{aligned}
$$

Using eq. (S35) in eq. (S30), one obtains:

$$
k_{\text {flash }}^{I}=k_{i}^{m}+\frac{k_{-t}^{m} \beta\left[H^{+}\right]}{k_{t}^{m}+\beta\left[H^{+}\right]}
$$

with

$$
\beta=\frac{k_{\text {out }} k_{-h}^{m}}{k_{\text {out }}+k_{h}^{m}}
$$

which are, respectively, eqs. (4A) and (4C) of the main text.

The fast component would be the fast equilibration between B-CTAB and Cc-CTAB:

$$
k_{\text {flash }}^{I I}=k_{t}^{m}+k_{-t}^{m}
$$

corresponding to eq. (4B) of the text. 
The two steady-state approximations are feasible if the tautomeric step is very fast and the decays are well separated events. Clearly this is not the case. Therefore only one steady-state approximation is made (eq. (S34)). Under such circumstances, the following set of differential equations is obtained:

$$
\begin{aligned}
& \frac{d[B-C T A B]}{d t}=k_{-t}^{m}[C c-C T A B]-\left(\beta\left[H^{+}\right]+k_{t}^{m}\right)[B-C T A B] \\
& \frac{d[C c-C T A B]}{d t}=-\left(k_{i}^{m}+k_{-t}^{m}\right)[C c-C T A B]+k_{t}^{m}[B-C T A B]
\end{aligned}
$$

The set can be solved according to the matrix method:

$$
\left|\begin{array}{cc}
-\left(\beta\left[H^{+}\right]+k_{t}^{m}\right)-\lambda & k_{-t}^{m} \\
k_{t}^{m} & -\left(k_{i}^{m}+k_{-t}^{m}\right)-\lambda
\end{array}\right|=0
$$

And the solutions are eqs. (5) of the text:

$$
\begin{aligned}
& k_{\text {flash }}^{I}=\frac{\left(k_{i}^{m}+k_{t}^{m}+k_{-t}^{m}+\beta\left[H^{+}\right)-\sqrt{\left(k_{i}^{m}+k_{t}^{m}+k_{-t}^{m}+\beta\left[H^{+}\right]\right)^{2}-4 k_{i}^{m} k_{t}^{m}-4\left(k_{i}^{m}+k_{-t}^{m}\right) \beta\left[H^{+}\right]}\right.}{2} \\
& k_{\text {flash }}^{I I}=\frac{\left(k_{i}^{m}+k_{t}^{m}+k_{-t}^{m}+\beta\left[H^{+}\right)+\sqrt{\left(k_{i}^{m}+k_{t}^{m}+k_{-t}^{m}+\beta\left[H^{+}\right]\right)^{2}-4 k_{i}^{m} k_{t}^{m}-4\left(k_{i}^{m}+k_{-t}^{m}\right) \beta\left[H^{+}\right]}\right.}{2}
\end{aligned}
$$




\section{References}

(1) Pina, F.; Maestri, M.; Balzani, V. in Handbook of Photochemistry and Photobiology; ASP, 2003;

Chapter 9, vol. 3, 411-449.

(2) Moncada, M.C.; Fernandéz, D.; Lima, J. C.; Parola, A. J.; Lodeiro, C.; Folgosa, F.; Melo, M. J.; Pina, F. Org. Biomol. Chem. 2004, 2, 2802-2808.

(3) Wünscher, H.; Haucke, G.; Czerney, P.; Kurzer, U.; J. Photochem. Photobiology, A: Chemistry, 2002, $151,75-82$.

(4) $\eta_{\mathrm{AH}+}$ is an efficiency independent on the quantum yield. 